\title{
What happened at Vienna's Allgemeines Krankenhaus after Semmelweis's contract as Assistant in the First Maternity Division was terminated?
}

\author{
P. P. JADRAQUE ${ }^{1 *}$ AND K. C. CARTER ${ }^{2}$ \\ ${ }^{1}$ Department of Epidemiology, Hospital General de La Palma, Breña Alta, Canarias 38710, Spain \\ ${ }^{2}$ Department of Philosophy (retired Professor.), Brigham Young University, Provo, UT 84602, USA
}

Received 5 December 2016; Final revision 16 March 2017; Accepted 30 March 2017;

first published online 2 May 2017

\section{SUMMARY}

Ignác Fülöp Semmelweis is famous for dramatically reducing puerperal mortality while he was an Assistant in Vienna's largest hospital, the Allgemeines Krankenhaus; he did this, mainly, by requiring medical personnel to disinfect their hands by washing in a chlorine solution. But Semmelweis was soon removed from his post as assistant. The conventional view, which is suggested by Semmelweis's own account, is that his contemporaries were skeptical of his results, that he was marginalized and that once he was no longer directly responsible for caring for maternity patients, puerperal mortality returned to its former high levels. In fact, the situation appears to have been quite different. In this paper, we calculate and discuss the number of deaths at the Allgemeines maternity clinic after Semmelweis was removed from his position. As we will see, his successors maintained a relatively low mortality rate roughly consistent with the rate Semmelweis himself achieved. This suggests that the chlorine washings were probably still used conscientiously after he left and that the opposition he encountered had other sources than doubts about the effectiveness of the chlorine washings.

Key words: Allgemeines Krankenhaus, Carl Braun, hand hygiene, hygiene and hospital infections, Semmelweis.

\section{INTRODUCTION}

In the late 18 th and early 19 th centuries, vast numbers of postpartum women died from a disease known as puerperal fever or childbed fever. In some maternity facilities, over a period of several years, mortality from this disease averaged more than twenty percent of all admitted patients [1]. In the maternity facilities of Vienna's main hospital, the Allgemeines Krankenhaus $(\mathrm{AKH})$, the overall mortality rate was relatively

\footnotetext{
* Author for correspondence: P. Jadraque, Department of Epidemiology, Hospital General de La Palma, 38710 Breña Alta Canarias, Spain.

(Email: pablojadraque@yahoo.es)
}

favourable - about $7-10 \%{ }^{1}$; however, there were about five times as many deaths in one part of the facility, called the First Division, as in another part, called the Second Division [2].

Ignác Fülöp Semmelweis is famous for dramatically reducing puerperal mortality in the First Division of the AKH. At the time, Semmelweis was Assistant to Johannes Klein who was Professor of Obstetrics in the First Division. Soon after being appointed, Semmelweis realized that, unlike the (female) student midwives in the Second Division, who did not conduct

1 There are many accounts of developments in the AKH maternity facility. Semmelweis's own account is in [2] (an accessible abridged translation is in [7]). Braun's account is in [3]. 
autopsies, (male) student obstetricians in the First Division regularly came to the obstetrical wards with hands reeking of decaying remains from the autopsies they were conducting in the morgue. He originally hypothesized that puerperal fever was caused by the cadaveric particles which could be identified by its smell - later he extended the cause to every decaying organic matter. Sometime in the middle of May 1847 (the exact date is unknown), Semmelweis began requiring all medical personnel to wash, thoroughly, in a chlorine solution, in order to deodorize the hands, before examining patients or delivering babies. Almost immediately, mortality in the First Division fell to about the same rate as was maintained in the Second Division [2].

Today, Semmelweis's views seem unquestionably correct. We now understand that decaying organic matter contains pathogenic flora and that washing with a chlorine solution can help disinfect the hands. Also, the deaths to admissions ratios at the AKH from the time it opened (1784) through the time Semmelweis supervised the use of chlorine washings (1849) are consistent with Semmelweis's statements. These ratios are shown in Fig. 1 below as slopes of the (straight, not drawn) lines that bind points. A horizontal line would mean there was no mortality, and an almost vertical line would indicate $100 \%$ mortality. In the period from 1784 until 1822, the ratios are small and, thus, the slopes are also. In 1823, Klein was named Professor and, in harmony with the growing interest in pathological anatomy, he introduced the dissection of cadavers - in this period the ratios grew abruptly and the slopes become correspondingly steep. In 1847, when Semmelweis introduced chlorine washings, the ratios fell and the slopes declined accordingly. In the Second Division, which opened in 1832 , the deaths to admissions ratios were similar to those in the First Division until students were separated by gender; at that point the ratios for the Second Division fell and those for the First Division rose.

Semmelweis' story is widely known, because, in spite of dramatically reducing mortality in his Division, Semmelweis encountered opposition at the AKH. In March of 1849, Semmelweis was removed from his position as Assistant in the First Division, and in May 1850 he gave a lecture on his experiences; the lecture was not well received. Semmelweis felt marginalized and, in October 1850, he abruptly left Vienna and returned to his native Budapest [2].

Semmelweis was replaced, as Assistant in the First Division, by Carl Braun, and, a few years later, in
1856, Braun succeeded Johannes Klein as Professor of Obstetrics. Throughout his career, Braun vigorously opposed Semmelweis's views about the etiology of childbed fever, and it is generally believed that, during his tenure, mortality in the First Division returned to a relatively high rate. This belief is based in part on Semmelweis's own claims about mortality at the AKH - Semmelweis wrote that Braun abandoned the practice of chlorine washings although he acknowledged that Braun recommended that students avoid examining women if their hands still smelled of cadavers [2]. But this information suggests that the relationship between Semmelweis and Braun may be more complex than one may initially suppose: Braun rejected some of Semmelweis's statements, but seems to have continued to enforce some of his practices. How is this to be explained?

Semmelweis calculated that, in comparison to the period before the adoption of chlorine washings, Braun's recommendation prevented 837 deaths between 1849 and 1853 and in $1858^{2}$, but, in comparison to the mortality rate he himself had achieved in 1848, Braun's leadership resulted in 317 preventable deaths. However, to our knowledge, no one has reliably analyzed the data to determine whether these results are accurate $^{3}$. Thus, the objectives of this paper are,

2 The results for 1858 are not directly relevant to those for the period we are investigating, but we include them because Semmelweis himself incorporates these results into those he provides for the earlier period. The yearly deaths to admissions ratio in 1858 is 2.05 cases per 100 admissions, not statistically different $(P>0.05)$ from the yearly deaths to admissions ratio in $1850,1.98$ cases per 100 admissions.

3 To date, the best discussion is found in Noakes TD et al. Semmelweis and the aetiology of puerperal sepsis 160 years on: an historical review. Epidemiology and Infection 2008;136(1):1-9. However, these authors, also, appear to have accepted Semmelweis's claims at face value without checking them carefully against his own data. They write that 'following the appointment of Carl Braun, mortality in both clinics at the AKH began to rise (Figs. 2 and 3 , line M)' (p. 7). But this is a mistake because, in their figures, the appointment of Braun is indicated by line L (not by line M) and their Fig. 3 shows, clearly, that mortality actually sank between lines L and $\mathrm{M}$ - thus, they appear to have misconstrued their own figure. Moreover, a closer look at their Fig. 3 suggests - we cannot assure because Supplementary materials do not include monthly data, that they did not use the whole data available after Semmelweis was substituted by Braun, those that we have used and are available on page 445 of Braun's book and consist of the values for every month from January, 1849 to December, 1852 (48 months), but a biased selection of 26 months selected by Semmelweis (not numbered Table on page 510 in Semmelweis's book), which consist of the data which meet two criteria: first, number of admissions is higher in the First Division than in the Second, and second and more important, mortality ratio is higher in the Second Division than in the First. If this is the case, it is not surprising that they make incorrect claims, such as this: 'Interestingly the mortality rate in clinic 1 remained lower than in clinic 2 between 1849 and 1852 even though Semmelweis was no longer present'. Because of this, and because of other mistakes, it appears to us 


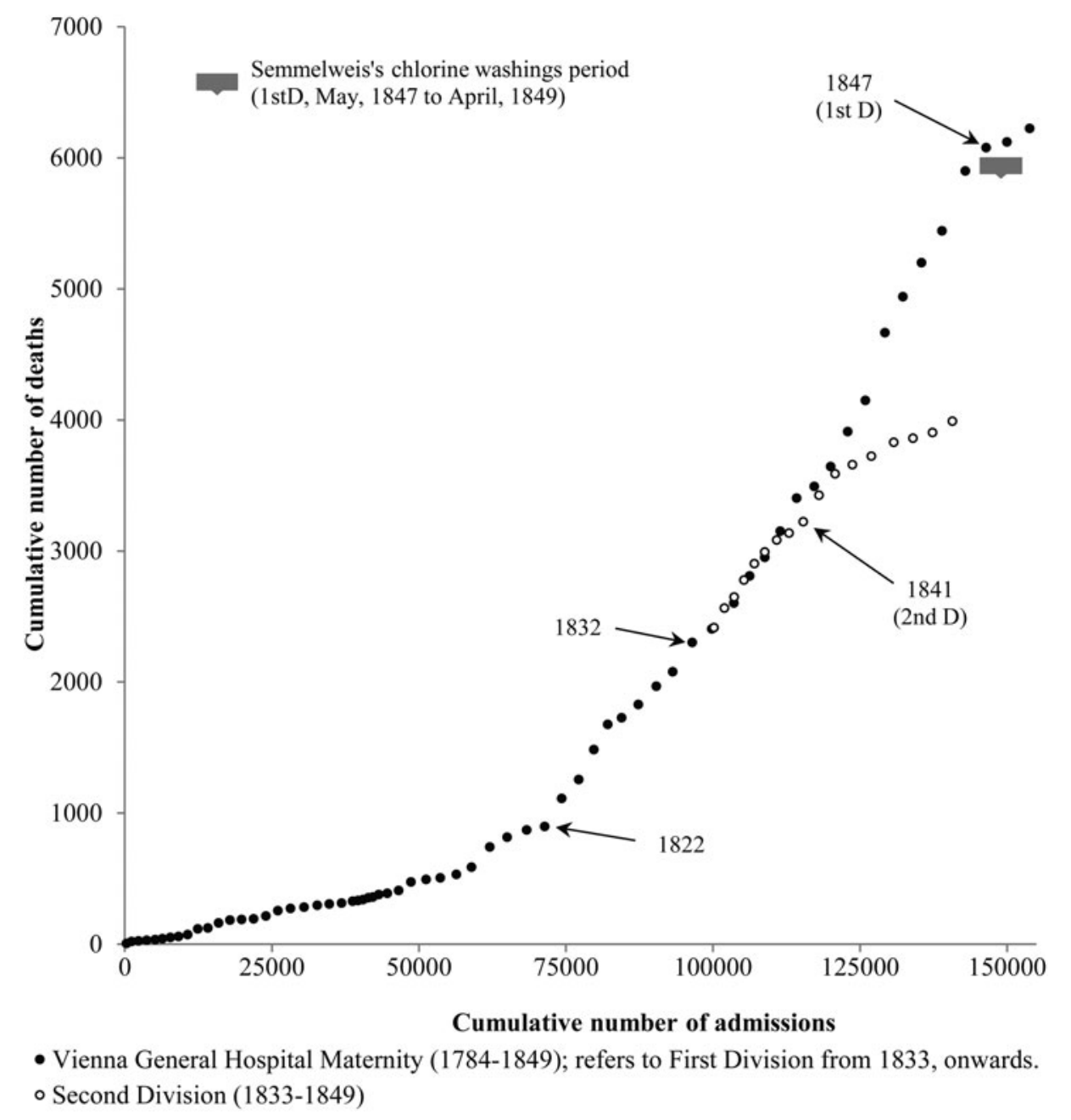

Fig. 1. Evolution of maternal death in the maternity facility at the Allgemeines Krankenhaus in Vienna for the period 1784-1849. Every point corresponds to one calendar year. 1st D refers to the First Division and 2nd D refers to the Second Division.

first, analyzing the epidemiological data for the periods before and after Semmelweis was removed from his post as Assistant to determine the actual mortality rates achieved by Semmelweis and Braun, and second, comparing and explaining some of the beliefs and practices that prevailed in the Viennese maternity facilities over this interval.

\section{METHODS}

Our calculations are based on data drawn from two sources: Semmelweis's main publication, Die Aetiologie, der Begriff und die Prophylaxis des Kindbettfirbers (1860) [2], and an obstetrical textbook, Klinik der Geburtshilfe und Gynaekologie (1855), which was

that everything these authors have said about maternal mortality at the AKH in the period following Semmelweis's removal from patient care is wrong or at least unwarranted. written by Carl Braun together with two of his Viennese colleagues and which reports and discusses events at the AKH maternity facility [3]. Semmelweis himself used Braun's monthly data in his own book and thus assumed that Braun's figures were correct.

We begin by calculating the cumulative deaths to admissions ratio by dividing the total number of deaths in the First Division of the AKH maternity facility by the total number of admissions for the period from June 1847 until March 1849 and multiplying by 100; these are the 22 months during which Semmelweis employed chlorine washings in the First Division. This ratio provides a base figure for comparison, and we refer to it as CMR-Ref. We calculate the expected number of deaths for any period by taking the product of CMR-Ref and the number of admissions in that period. We calculate the theoretically 
preventable deaths by subtracting the expected number of deaths from the observed number of deaths. The excess percentage of preventable deaths is obtained by dividing the number of theoretically preventable deaths by the expected number of deaths. Values enclosed in parentheses indicate the $95 \%$ confidence limits; the $t$ test is used to identify limits with statistical significance and to compare values.

\section{RESULTS}

Over the 22 months during which Semmelweis supervised the First Division, 6495 women were admitted and 142 died of puerperal fever. Thus, as explained in the previous paragraph, the cumulative deaths to admissions ratio (CMR-Ref) is $2.19(1.78 ; 2.59)$ cases per 100 admissions.

From April 1849 until December 1853, while the First Division was under Braun's leadership, there was a total of $66(11 ; 122)$ preventable deaths in the First Division beyond what would be expected given the results obtained when chlorine washes were monitored by Semmelweis $(P<0 \cdot 05)$. However, these 66 preventable deaths over a period of almost 5 years 13 per year on average - are approximately 33 times smaller than the number of preventable deaths $2208(2156 ; 2260)$ - that one would expect had Braun allowed puerperal mortality to have returned to the high levels of 1846 when the yearly deaths to admissions ratio was $13 \cdot 69 \%$.

Excepting 1853, for which monthly data are not available, Fig. 2, below, shows monthly mortality figures from January, 1846, until the end of Braun's tenure at the facility (1853). As in Fig. 1, the slope of the (straight, not drawn) line connecting mortality figures is the observed deaths to admissions ratio. Projections of some ratios are disclosed as drawn lines. As one sees, the mortality rates under Braun's leadership were quite similar to those during Semmelweis's leadership and are much smaller than those from the period before the use of chlorine washings.

The figure also shows that Semmelweis's calculations are misleading. To be consistent, if Semmelweis adopts as his standard the results he achieved in his own best partial period (1848), he should also have chosen for comparison the results from Braun's best partial period (April, 1850 to March, 1851) rather than the results from Braun's entire tenure. And, if we compare these figures (Semmelweis: 3456 admissions and 45 deaths; yearly deaths to admissions ratio of $1 \cdot 30$ cases per 100 admissions, and Braun: 3810 admissions and 45 deaths; yearly ratio of $1 \cdot 18$ cases per 100 admissions) statistically, the mortality under Braun's supervision was not significantly different $(P>0.05)$ from the mortality under Semmelweis's leadership. Moreover, if we take, as the standard for comparison, not CMR-Ref (the cumulative deaths to admissions ratio for Semmelweis's entire time supervising the chlorine washings) but rather the ratio merely for 1848 (Semmelweis's standard), then over the whole time that Semmelweis used chlorine washings, he himself had $57(37 ; 78)$ excessive deaths, that is, his mortality rate was 1.4 times that maintained in his best period a $40 \%(22 \% ; 67 \%)$ excess percentage of preventable deaths.

\section{DISCUSSION}

Our results show that Braun maintained almost exactly the same low mortality as Semmelweis and therefore strongly suggests that, as Braun himself claimed, during his tenure in the First Division, Braun continued, assiduously, to require the chlorine washings - in fact, Braun stated that, under his supervision, between 1849 and 1852, chlorine washes were monitored very strictly even in the Second Division, where chlorine washings were not performed while Semmelweis was Assistant [3, p. 473]. Pathogenic flora is necessary for puerperal sepsis but it is certainly not sufficient. Many other factors - for example the relative virulence of the infecting strain of pathogenic organism, the differing susceptibility of patients, even changes in the personnel and staff providing care can all contribute to determining whether a given patient will become diseased and, if so, how mild or serious the case may be. Thus, the use of chlorine washings is certainly not the only factor that would have influenced mortality rates during the period we are considering. However, it was not possible to assess these other variables.

There is no doubt that Semmelweis encountered serious opposition at the AKH. If, as Braun insisted and as we have just argued, chlorine washings continued to be used, in force, after Semmelweis was dismissed, what was the source of this opposition? Three quite different sources have already been identified and discussed in the literature, and, having ruled out skepticism about the effectiveness of the chlorine washings, our conclusion is that a combination of these likely provides the best explanation: first, Semmelweis's tenure in the First Division took place during the tumultuous revolutions that swept 


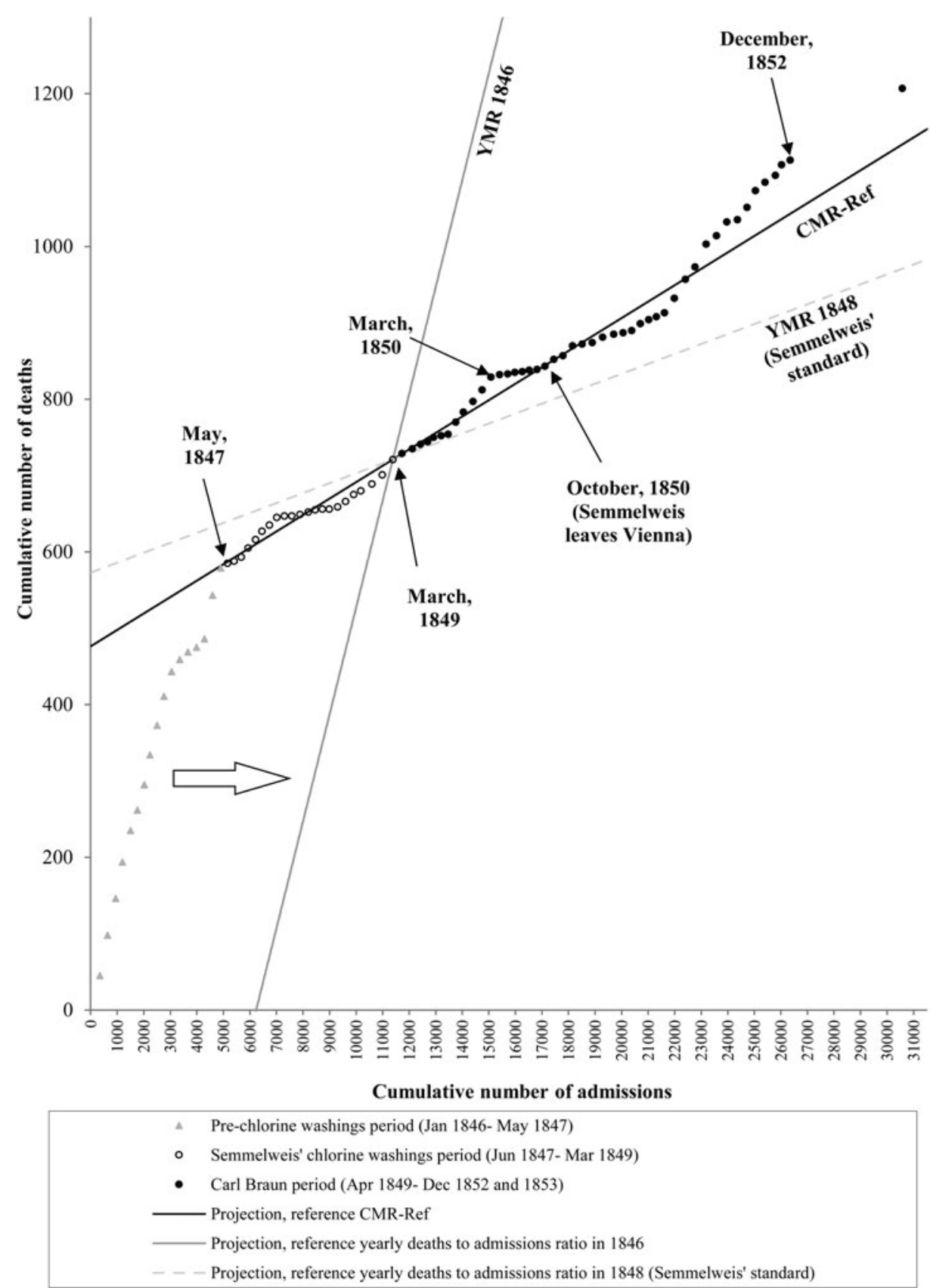

Fig. 2. First Division of the Maternity Facility of the AKH from January 1846 to December 1853. Every point corresponds to one calendar month, except for the last one, which corresponds to a calendar year (1853). Lines that show expected values (projections) are shifted to cross over the point that indicates the values for March, 1849. YMR refers to yearly deaths to admissions ratio.

Europe in 1848. In the Habsburg empire, which centered in Vienna and which included much of central Europe, these revolutions involved major nationalist uprisings by the Hungarians and the Bohemians. Although Semmelweis was ethnically German, he was born and raised in Budapest and always identified himself as a Hungarian. His own political sympathies are unknown [4], but he may certainly have been viewed with some suspicion by his chief, Johannes Klein, who was a native Austrian and was closely allied with the ruling Habsburgs. These background issues are relatively obvious and, having been discussed for decades, do not constitute a novel explanation of anything. However, the political tensions at the AKH had more immediate sources than vague concerns about nationalistic aspirations throughout 
the Habsburg empire. Some years ago, Erna Lesky demonstrated that, while Semmelweis worked in the First Division, he was inadvertently drawn into a power struggle between the younger members of the medical faculty, including such persons as Josef Skoda and Karl Rokitansky (both of whom happen to have been native Bohemians), and the senior faculty members including especially Semmelweis's own chief, Johannes Klein [5]. The evidence is that, without necessarily understanding or really agreeing with Semmelweis, in their quest to gain greater independence and influence at the medical school, these younger members of the faculty used Semmelweis's success as a weapon to discredit and to undermine Klein and his peers ${ }^{4}$. Thus, given this situation in the medical school, especially when viewed against the general background of nationalistic uprisings throughout the Empire, it cannot be surprising that, on political grounds alone, Klein may have been eager to rid himself of the young Semmelweis.

However, while this may help us understand Klein's response to Semmelweis, it does not explain how Braun could, at once, adopt the chlorine washings and yet argue consistently and bitterly against Semmelweis. The best explanation for this apparent inconsistency seems to be Semmelweis's insistence on what he himself identified as his central claim, namely, that childbed fever invariably had one and only one cause: decaying organic matter. Indeed, probably in his 1850 lecture, but certainly by 1860 , when he published his book, for Semmelweis this had become a matter of definition - he literally defined 'childbed fever' as 'a resorption fever dependent on the resorption of decaying animal-organic matter' [2, p. 102]. But, at the time, diseases were almost universally conceived of as collections of symptoms or as specific pathological modifications, and given such definitions, most diseases were believed, correctly, to have wide ranges of possible causes [6]. Thus, to contemporary physicians, Semmelweis's claim and the definition based on it were almost inconceivable - the definition verged on nonsense. It was as though Semmelweis excluded, by definition, nearly all cases of childbed fever except those that could be controlled by chlorine

\footnotetext{
4 After Semmelweis left Vienna, neither Skoda nor Rokitansky ever mentioned Semmelweis in lectures or published works - not even in discussions of childbed fever [7, p. 52]. It is impossible to reconcile this fact with the conventional view that these professors agreed with and were supportive of Semmelweis's innovation. The most likely explanation is that they simply failed to see the value or the justification for Semmelweis's conception of childbed fever.
}

washings ${ }^{5}$. Thus, one early critic complained that Semmelweis's solution to the problem of childbed fever was nothing more than a verbal trick - something he labeled, correctly, an Egg of Columbus [2, p. 223]. Not surprisingly, through 1860 , regardless of whether they accepted the chlorine washings, everyone who responded to Semmelweis either rejected his central claim or failed to understand exactly what he was maintaining [7].

So what were Braun's views about the etiology of childbed fever and the use of chlorine washings? In full harmony with the medical thinking of the time, Braun identified 30 possible causes of childbed fever [3, pp. 450-487]. The 28th cause in Braun's list was 'cadaverous infection'. Braun observed that, according to Semmelweis, this was 'almost the only cause of puerperal fever epidemics' and Braun devoted nearly one-third of his discussion of the etiology of the disease to an extensive consideration of this one possible cause and of ways of avoiding it [3, pp. 465-477]. After a review of the literature and a report of his own experiences with chlorine washings, Braun admitted that the washings could be useful against this one possible cause. However, he observed that the washings 'could not be trusted blindly' and that 'any student should be strictly prohibited from examining pregnant or delivering women if, on the same day, he has contacted cadavers' [3, p. 473]. In a later publication, Braun considered the same 30 possible causes of childbed fever. In this later discussion (in which Semmelweis is not mentioned by name), after expressing doubt that contact with cadavers could be a prominent cause of childbed fever in maternity clinics, Braun added 'however, we would regard it as the height of irresponsibility if, even after carefully cleaning, one were to allow or to personally undertake an examination or an operation on pregnant, delivering, or postpartum women if one could still detect the smell of cadavers on the hands of the agent conducting the examination' [8]. So Braun was able to accept and adopt the chlorine washings as one tool against this one possible cause of childbed fever while at the same time emphatically opposing Semmelweis's central claim that this was the only possible cause of the disease. No doubt, in addition to the chlorine washings, his policy of preventing contact between

5 Semmelweis acknowledged that, in some cases, childbed fever originated from decaying organic matter located in the birth canal of the delivering woman - in such cases, while such cases still satisfied his definition of the disease, he saw no hope of controlling such cases by chlorine washings. 
patients and persons whose hands still reeked of cadavers and even his installation of improved ventilation could also have contributes to his success in avoiding the disease. This helps us understand that Braun's position was more nuanced than a cursory view might lead one to believe. It also helps us see how Semmelweis's unorthodox conception of childbed fever contributed to the hostility he encountered from physicians at the AKH. As Theodore G. Oberchain has observed, 'in his era, 30 years before the idea of bacteria was generally appreciated and the germ theory accepted as fact, [the concept of] 'one agent per one disease' was just too revolutionary to be acceptable' [9].

A third possible source of opposition to Semmelweis has been identified and must be taken into account. In 2003, Sherwin Nuland advanced the notion that, from the very start, Semmelweis presented his ideas with such 'passionate arrogance' that he offended virtually all of his associates [10]. The inevitable result was rejection. Thus, on this account, Semmelweis himself was ultimately responsible for the opposition he encountered at the AKH Nuland writes that 'he brought it on himself' [10]. This is not the place for a thorough examination of this rather surprising conceit. Looking back nearly 200 years it is difficult to weigh the possible influence that Semmelweis's personality may have had in generating the opposition he encountered. The only evidence of Semmelweis's personality we now have is isolated comments by contemporaries. In his recent study of the life and work of Semmelweis, certainly the most thorough and the most scholarly one that yet appeared, Theodore Obenchain considers Nuland's argument 'point by detailed point' and ultimately rejects Nuland's conclusions [9, p. 220]. Obenchain argues persuasively that Semmelweis suffered from manic-depressive illness but that the symptoms of this disorder did not become apparent until between about 1857 and 1860 - so roughly 10 years after Semmelweis left the AKH. Obenchain reviews the evidence regarding Semmelweis's personality while he was working at the AKH. This evidence can be summarized in the words of one contemporary medical student and friend who said this of Semmelweis: 'in short he was a jolly companion; you could not wish for a better one' [9, p. 63]. In independent publications that appeared in 1882, not mentioned by Obenchain, two other colleagues from the AKH period referred to him as 'the genial Semmelweis' [11]. Thus, while it is conceivable that Semmelweis offended the professors by presenting his views too forcefully, given what was written of his personality in the AKH period, to us seems most unlikely that passionate arrogance was a significant issue.

\section{SUPPLEMENTARY MATERIAL}

The supplementary material for this article can be found at https://doi.org/10.1017/S0950268817000875

\section{ACKNOWLEDGEMENTS}

The views presented in this article are those of the authors and do not necessarily represent the opinions of their affiliated institutions.

\section{DECLARATION OF INTEREST}

None.

\section{REFERENCES}

1. DeLacy M. Puerperal fever in eighteenth-century Britain. Bulletin of the History of Medicine [Internet]. 1989 Jan [cited 2 December 2016]; 63(4): 521-56 (http://www.ncbi.nlm.nih.gov/pubmed/2696569).

2. Semmelweis IF. Die Aetiologie, der Begriff und die Prophylaxis des Kindbettfiebers [Internet]. 1861 [cited 23 August 2016] (https://books.google.com/books?id= O2haAAAAcAAJ\&pgis=1).

3. Chiari JB, Braun C, Spaeth J. Klinik der Geburtshilfe und Gynaekologie [Internet]. Ferdinand Enke; 1855 [cited 23 August 2016]. 738p (https://books.google. com/books?id=F8REAAAAcAAJ\&pgis=1).

4. Carter KC, Carter BR. Childbed Fever: a Scientific Biography of Ignaz Semmelweis. Westport, Connecticut: Greenwood, 1994, p. 58.

5. Lesky E. Ignaz Philipp Semmelweis und die Wiener Medizinische Schule [Internet]. Böhlau; 1964 [cited 2 December 2016]. 93p (https://books.google.com/books? $\mathrm{id}=\mathrm{H13}$ _vQAACAAJ\&pgis $=1$ ).

6. Carter KC. The Rise of Causal Concepts of Disease: Case Histories [Internet]. Ashgate; 2003 [cited 2 December 2016]. 237p (https://books.google.com/ books?id=umRrAAAAMAAJ\&pgis $=1$ ).

7. Carter KC. The Etiology, Concept, and Prophylaxis of Childbed Fever [Internet]. University of Wisconsin Press; 1983 [cited 23 August 2016]. 263p (https:// books.google.com/books?id=hnezngRghTgC\&pgis=1).

8. Lehrbuch der Geburtshülfe: mit Einschluss der operativen Therapeutik, der...- Carl Rudolf Braun (Ritter von Fernwald) [Internet]. Wien; 1857 [cited 22 February 2017]. 1014p. (https://books.google.es/books/about/ Lehrbuch_der_geburtshülfe.html?id=iLItMQAACAAJ \&redir_esc $=$ y).

9. Obenchain TG. Genius belabored: childbed fever and the tragic life of Ignaz Semmelweis [Internet]. The 
University of Alabama Press; 2016 [cited 22 February 2017] (https://muse.jhu.edu/book/46875).

10. Nuland SB. The Doctors' Plague: Germs, Childbed Fever, and the Strange Story of Ignác Semmelweis. New York: W.W. Norton, 2004, 205p.
11. Carter KC, Kay C. The Decline of Therapeutic Bloodletting and the Collapse of Traditional Medicine [Internet]. Transaction Publishers; 2012 [cited 22 February 2017]. 160p (https://books.google.es/books? $\mathrm{id}=7 \mathrm{kOvJqoEwpcC} \&$ printsec $=$ frontcover $\& \mathrm{hl}=\mathrm{es} \# \mathrm{v}=$ onepage $\& \mathrm{q} \& \mathrm{f}=$ false). 